\title{
Anti-ceramide antibody prevents the radiation gastrointestinal syndrome in mice
}

\author{
Jimmy Rotolo,, ${ }^{1}$ Branka Stancevic, ${ }^{1}$ Jianjun Zhang, ${ }^{1}$ Guoqiang Hua, ${ }^{1}$ John Fuller, ${ }^{1}$ Xianglei Yin, ${ }^{1}$ \\ Adriana Haimovitz-Friedman, ${ }^{2}$ Kisu Kim, ${ }^{3}$ Ming Qian, ${ }^{3}$ Marina Cardó-Vila, ${ }^{3}$ Zvi Fuks, ${ }^{2}$ \\ Renata Pasqualini, ${ }^{3}$ Wadih Arap, ${ }^{3}$ and Richard Kolesnick ${ }^{1}$
}

${ }^{1}$ Laboratory of Signal Transduction and ${ }^{2}$ Department of Radiation Oncology, Memorial Sloan-Kettering Cancer Center (MSKCC), New York, New York, USA. ${ }^{3}$ David H. Koch Center, The University of Texas MD Anderson Cancer Center, Houston, Texas, USA.

\begin{abstract}
Radiation gastrointestinal (GI) syndrome is a major lethal toxicity that may occur after a radiation/nuclear incident. Currently, there are no prophylactic countermeasures against radiation GI syndrome lethality for first responders, military personnel, or remediation workers entering a contaminated area. The pathophysiology of this syndrome requires depletion of stem cell clonogens (SCCs) within the crypts of Lieberkühn, which are a subset of cells necessary for postinjury regeneration of gut epithelium. Recent evidence indicates that SCC depletion is not exclusively a result of DNA damage but is critically coupled to ceramide-induced endothelial cell apoptosis within the mucosal microvascular network. Here we show that ceramide generated on the surface of endothelium coalesces to form ceramide-rich platforms that transmit an apoptotic signal. Moreover, we report the generation of 2A2, an anti-ceramide monoclonal antibody that binds to ceramide to prevent platform formation on the surface of irradiated endothelial cells of the murine GI tract. Consequently, we found that $2 \mathrm{~A} 2$ protected against endothelial apoptosis in the small intestinal lamina propria and facilitated recovery of crypt SCCs, preventing the death of mice from radiation GI syndrome after high radiation doses. As such, we suggest that $2 \mathrm{~A} 2$ represents a prototype of a new class of anti-ceramide therapeutics and an effective countermeasure against radiation GI syndrome mortality.
\end{abstract}

\section{Introduction}

Characterized clinically by anorexia, vomiting, diarrhea, dehydration, systemic infection, and, in extreme cases, septic shock and death, the radiation gastrointestinal (GI) syndrome involves destruction of crypt/villus units, loss of mucosal integrity, and infection by resident enterobacterial flora (1-3). While conventional radiobiology considers unrepaired or misrepaired DNA doublestrand breaks in stem cell clonogens (SCCs) as autonomous lesions leading to irreversible tissue injury, our recent studies have challenged this paradigm, presenting genetic evidence that acute endothelial damage also plays a major role in GI tract injury (4-6). Within minutes of radiation exposure, endothelial acid sphingomyelinase (ASMase) is activated, catalyzing ceramide generation on the external plasma membrane of mouse and human endothelium to initiate apoptotic signaling $(7,8)$. Endothelium displays 20 -fold more ASMase than other mammalian cells, almost exclusively in a secretory form, which makes them particularly vulnerable to ceramide-induced apoptosis $(9,10)$. Early evidence indicates that vascular compromise, consequent to endothelial cell apoptosis, impairs radiation-injured SCC DNA damage repair, resulting in SCC demise. In several mouse strains, endothelial apoptosis occurs between 8 and 15 Gy $(4,6)$, which encompasses doses that cause both sublethal ( $\leq 14 \mathrm{~Gy}$ ) and lethal ( $\geq 15 \mathrm{~Gy}$ ) GI tract injury (5), beginning at 1 hour and peaking at 4 to 6 hours after irradiation $(4,6,11)$. Attenuation of intestinal endothelial apoptosis

Authorship note: Renata Pasqualini, Wadih Arap, and Richard Kolesnick contributed equally to this work.

Conflict of interest: Wadih Arap and Renata Pasqualini have equity positions in Mercator Therapeutics and Ablaris Therapeutics. University of Texas manages terms of these arrangements in accordance with its conflict of interest policy.

Citation for this article: J Clin Invest. 2012;122(5):1786-1790. doi:10.1172/JCI59920. by genetic inactivation of ASMase-mediated ceramide generation enhances SCC survival, facilitating repair of crypt damage and rescue of animals from GI lethality $(4,6)$. These observations provide the basis for developing a neutralizing anti-ceramide monoclonal antibody as a potential radiation countermeasure.

\section{Results and Discussion}

Initial studies examined whether radiation-induced ceramide locally reorganizes endothelial plasma membranes to form ceramide-rich platforms (CRPs), sites of ceramide-mediated transmembrane signal transmission for diverse stresses in other mammalian cell types (7). These studies used bovine aortic endothelial cells (BAECs), as previous reports detail ionizing radiation activation of the ASMase apoptotic program in these cells (12-14). In this study, ionizing radiation (10 Gy) induced a rapid increase in BAEC ASMase enzymatic activity from a baseline of $171 \pm 5$ $\mathrm{nmol} / \mathrm{mg} / \mathrm{h}$ to a peak of $307 \pm 24 \mathrm{nmol} / \mathrm{mg} / \mathrm{h} 1.5$ minutes after stimulation $(P<0.005$ vs. unirradiated control; Supplemental Figure 1A; supplemental material available online with this article; doi:10.1172/JCI59920DS1). Concomitantly, cellular ceramide increased from $157 \pm 12 \mathrm{pmol} / 10^{6}$ cells to $203 \pm 10 \mathrm{pmol} / 10^{6}$ cells ( $P<0.01$ vs. unirradiated control) within 1 minute of stimulation and persisted for over 2 minutes before decreasing toward baseline (Supplemental Figure 1B). Simultaneous increase of neutral sphingomyelinase or ceramide synthase activity was not detected (data not shown), confirming radiation-induced ceramide generation as ASMase mediated. At the same time, cell surface platforms enriched in ASMase and ceramide were observed by fluorescence microscopy (Figure 1A). Formation of CRPs, identified as ceramide clustered into cell surface macrodomains of $500 \mathrm{~nm}$ up to several microns (7), was detected as early as 30 seconds after irradiation, peaking at 1 minute with $32 \% \pm 2 \%$ of the population 

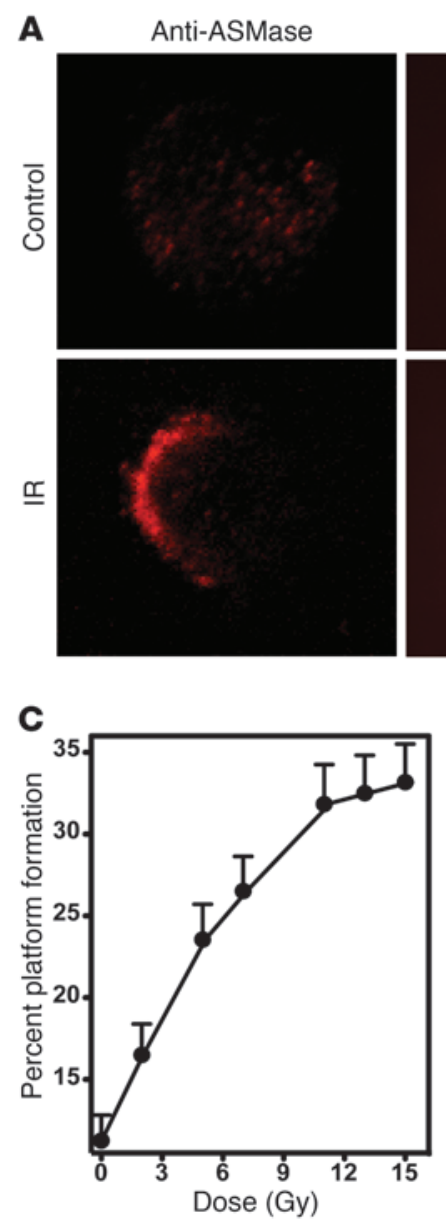

Anti-ceramide

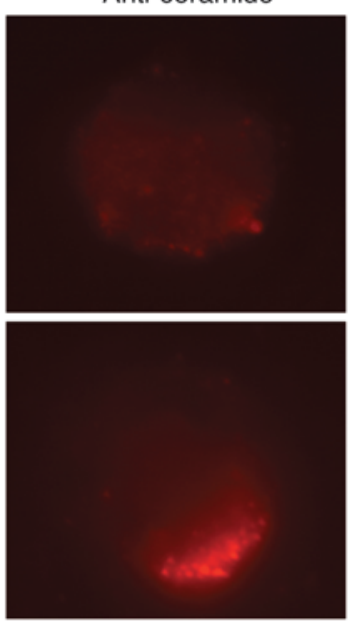

D

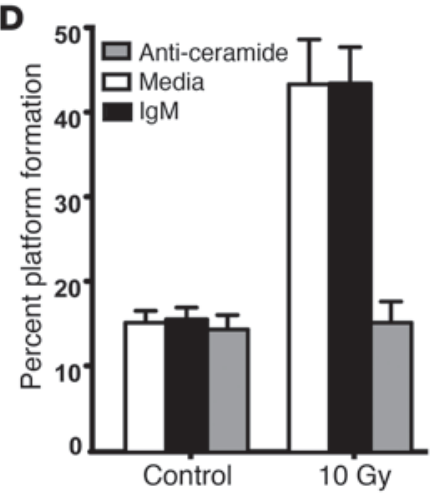

B

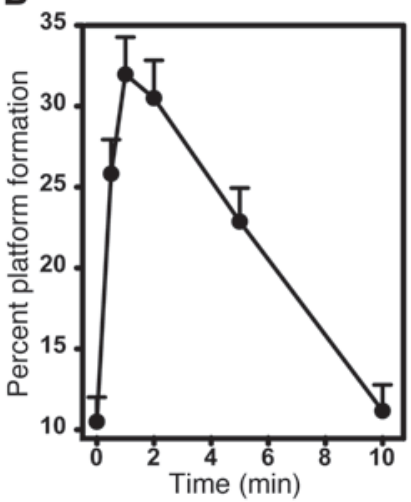

Figure 1

CRPs regulate ionizing radiation-induced apoptosis of BAECs. (A) Cells were fixed 1 minute after 10 Gy, and platforms were identified by fluorescence microscopy after staining with anti-ceramide IgM MID 15B4 (1:50 dilution; Alexis Biochemicals) or rabbit anti-ASMase antibody 1598 (1:100 dilution), followed by Cy3-conjugated anti-mouse or anti-rabbit IgM (1:500 dilution; Roche), respectively. Images (original magnification, $\times 400$ ) are each representative of 3 experiments, in which more than 100 cells were analyzed. IR, ionizing radiation. (B and C) Time- and dosedependent platform generation in BAECs. Cells were stimulated with (B) 10 Gy for indicated times or (C) increasing radiation doses for 1 minute and stained with anti-ceramide antibody, and platforms were identified as in A. (D and E) BAECs were pretreated with anti-ceramide MID 15B4 $(1 \mu \mathrm{g} / \mathrm{ml})$. (D) Platforms were detected after staining with rabbit anti-ASMase antibody 1598 as in A, while (E) apoptosis was quantified by fluorescent microscopy following $10 \mathrm{~Gy}$ after fixing and staining with Hoechst-33258 (12). (B-E) Data (mean $\pm 95 \%$ confidence limits) are collated from 3 experiments using 400 cells per point.

exhibiting platforms $(P<0.001$ vs. unstimulated control; Figure 1B). Platform formation was dose dependent at 1 minute, reaching a maximum at $11 \mathrm{~Gy}(P<0.001)$, with an $\mathrm{ED}_{50}$ of approximately 5 Gy (Figure $1 C)$. This dose range is virtually identical to that published for induction of radiation-induced apoptosis in BAECs (15). Preincubation of BAECs with MID 15B4 (a commercially available anti-ceramide antibody), a strategy that neutralizes cell surface ceramide and blocks ceramide-induced coalescence in other cell types (16-19), inhibited radiation-induced formation of CRPs (Figure 1D). CRPs were observed in $43 \% \pm 6 \%$ of BAECs pretreated with irrelevant $\operatorname{IgM}(P<0.01$ vs. nonirradiated control), whereas pretreatment with the anti-ceramide antibody MID 15B4 $(1 \mu \mathrm{g} / \mathrm{ml})$ reduced CRPs to $16 \% \pm 2 \%$ of the population, which was not significantly different from the baseline $15 \% \pm 3 \%$ in unirradiated cells $(P>0.1)$. Surface ceramide neutralization and CRP inhibition attenuated $10 \mathrm{~Gy}$-induced apoptosis by $71 \%$ for up to 8 hours after stimulation (Figure $1 \mathrm{E} ; P<0.001$ ). Similar anti- ceramide antibody inhibition of radiation-induced (5-20 Gy) CRP formation and apoptosis were observed in Jurkat $\mathrm{T}$ lymphocytes (Zhang and Kolesnick, unpublished observations).

Based on these observations, we generated a mouse monoclonal anti-ceramide IgM, termed 2A2, with specific affinity for ceramide compared with phosphorylcholine, diacylglycerol, dihydroceramide, sphingomyelin, or GM1, as determined by ELISA (Supplemental Figure 2). 2A2 inhibited CRP formation and ceramide-mediated apoptosis (data not shown) and dose dependently inhibited endothelial cell apoptosis in vivo. Intravenous $2 \mathrm{~A} 2$ administration $(1,000 \mu \mathrm{g} / 25 \mathrm{~g}$ mouse $)$ to $\mathrm{C} 57 \mathrm{BL} / 6$ mice 15 minutes prior to the $\mathrm{LD}_{100}$ of $15 \mathrm{~Gy}$ whole-body irradiation (WBI) reduced peak endothelial apoptosis within the lamina propria microvasculature by $83 \%$, from a mean of $9.2 \pm 0.5$ apoptotic endothelium per villus to $1.6 \pm 0.3$ apoptotic endothelium per villus (Figure $2 \mathrm{~A} ; P<0.05$ ). Thus, $2 \mathrm{~A} 2$ phenocopies the genetic inhibition of radiation-induced intestinal endothelial apoptosis 
A
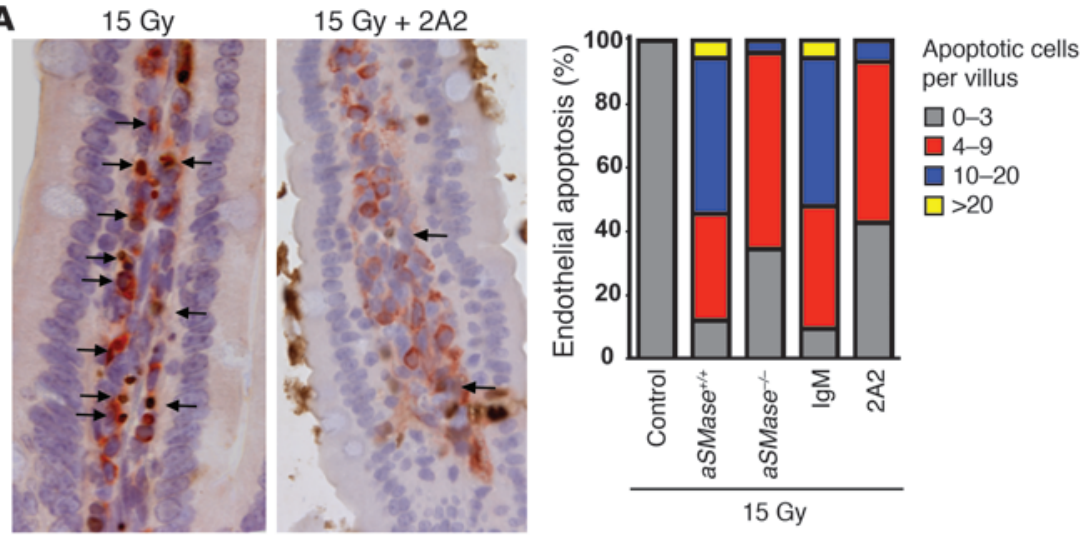

B

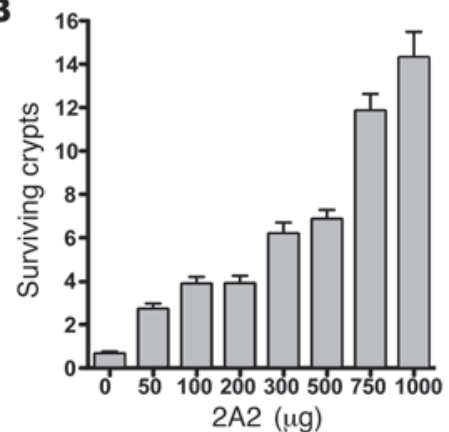

C

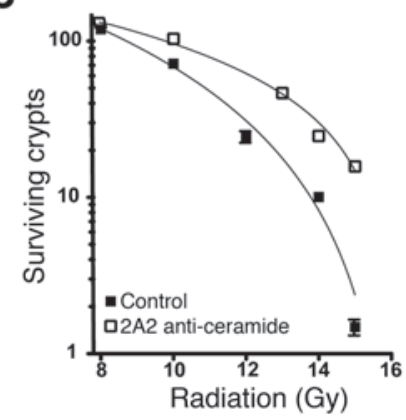

Figure 2

2A2 antibody inhibits radiation-induced endothelial apoptosis and crypt lethality. (A) Purified 2A2 or irrelevant IgM control $(1,000 \mu \mathrm{g})$ was injected intravenously into C57BL/6 mice 15 minutes prior to $15 \mathrm{~Gy} \mathrm{WBI}$, and proximal jejunum was harvested 4 hours thereafter. Endothelial apoptosis was identified by microscopic detection of TUNEL (brown) and CD34 (red) double-positive endothelium (indicated by arrows; original magnification, $\times 400$ ). Data, compiled from 3 experiments using 3 mice each analyzing 200 intact villi per mouse, are presented as a histogram. Note that approximately half of the villi in wild-type mice display 10 or more apoptotic endothelial cells after irradiation, effectively abrogated by preventing

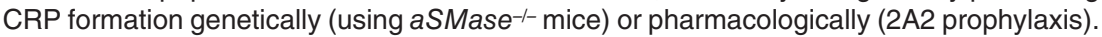
(B) 2 A2 dose dependently protects small intestinal crypts. C57BL/6 mice were administered purified $2 \mathrm{~A} 2(0-1,000 \mu \mathrm{g}) 15$ minutes prior to $15 \mathrm{~Gy} \mathrm{WBI}$. (C) Administration of 2A2 $(1,000 \mu \mathrm{g}) 15$ minutes prior to 8 to $15 \mathrm{~Gy}$ WBI enhances crypt survival, quantified by the microcolony assay of Withers and Elkind. (B and C) Data (mean \pm SEM) are compiled from 3 experiments of 2 mice each, analyzing 10-20 intestinal circumferences per mouse.

conferred by ASMase deletion in aSMase ${ }^{-/}$mice (Figure 2A, right). Consequently, intravenous $2 \mathrm{~A} 2$ increased crypt survival, as quantified by the microcolony assay of Withers and Elkind (Figure 2, $\mathrm{B}$ and $\mathrm{C}$ ), widely considered the gold standard assay for assessing injury to the GI tract stem cell compartment (20). The number of surviving crypts per intestinal circumference was reduced from $149.9 \pm 1.1$ in unirradiated animals to $1.4 \pm 0.2$ after 15 Gy (Figure 2B). As little as $50 \mu \mathrm{g} 2 \mathrm{~A} 2$ antibody/25 g mouse increased the number of surviving crypts $(P<0.05)$, while maximal protection was achieved with $1,000 \mu \mathrm{g} 2 \mathrm{~A} 2$ antibody $/ 25 \mathrm{~g}$ mouse. $2 \mathrm{~A} 2$ afforded protection when delivered within 2 hours preceding irradiation, with minimal protection for up to 24 hours (data not shown). ASMase-mediated ceramide was confirmed to be the target for this protection, as $2 \mathrm{~A} 2$ did not improve crypt survival in aSMase $e^{-/}$mice (Supplemental Figure 3). 2A2 protection against ceramide-mediated endothelial apoptosis enhanced crypt survival within the range of 8 to $15 \mathrm{~Gy}$ (Figure 2C), and animals adminis- tered $2 \mathrm{~A} 2$ preceding irradiation with 15 Gy displayed $15.8 \pm 1.0$ surviving crypts per intestinal circumference $(P<0.001 ; n=$ a minimum of 52 circumferences scored per radiation dose). Numerous studies indicate that this level of surviving crypts after irradiation is consistent with small intestinal regeneration and mouse survival $(1,20)$. Ex vivo irradiation of cultured crypts supports endothelium as the target for the protective effects of $2 \mathrm{~A} 2$, as no impact on crypt epithelial apoptosis was observed (Supplemental Figure 4). 2A2 administration was without toxicity, as complete histologic examination of unirradiated animals receiving $2 \mathrm{~A} 2$ did not display pathology.

To establish whether $2 \mathrm{~A} 2$ protects against radiation GI syndrome lethality, mice were administered 2A2 plus hematopoietic stem cell transplant (HSCT) to prevent BM aplasia. Note that in control studies $2 \mathrm{~A} 2$ did not protect against BM lethality occurring at radiation doses of less than $10 \mathrm{~Gy}$ (data not shown). Here, we report that 2A2-treated mice displayed increased 90 -day survival from $0 \%$ to $80 \%$ after administration of $15 \mathrm{~Gy}$ (Figure 3A; $P<0.01$ ), whereas control IgM did not impact survival (Figure 3A). Necropsy revealed that the $20 \%$ of 2A2-treated animals that had succumbed to radiotoxicity died with fully regenerated intestinal mucosa (Figure 3B), virtually identical to those that were rescued. Marrows of these mice, however, were extensively depleted, indicating graft failure despite HSCT, presumably due to BM stromal damage (Supplemental Figure 5). Thus, $100 \%$ of animals receiving $2 \mathrm{~A} 2$ were saved from radiation GI syndrome lethality at $15 \mathrm{~Gy}$. In contrast, $100 \%$ of animals receiving irrelevant IgM (isotype control) or no antibody (vehicle only) consistently died with denuded intestinal mucosa (Figure 3B) and clinical evidence of radiation GI syndrome lethality. Similar results were obtained in mice treated with $2 \mathrm{~A} 2$ prior to administration of $16 \mathrm{~Gy}$, as 90 -day survival was $60 \%$ (Figure 3A) and necropsies revealed that the $40 \%$ of mice that succumbed had normally regenerative GI tracts and $\mathrm{BM}$ transplant failure. At the $\mathrm{LD}_{50}$ dose of $14 \mathrm{~Gy}, 2 \mathrm{~A} 2$ plus HSCT saved $100 \%$ of animals (Supplemental Figure $6 \mathrm{~A}$ ). Even at the very high radiation dose of $17 \mathrm{~Gy}, 2 \mathrm{~A} 2$ protected $50 \%$ of GI tracts, as $25 \%$ of the $2 \mathrm{~A} 2$-pretreated mice survived ad infinitum (Supplemental Figure $6 \mathrm{~B}$ ), and $33 \%$ of those that succumbed died with intact GI tracts. To confirm that HSCT did not contribute to the protective effects of $2 \mathrm{~A} 2$, a subtotal body irradiation model was used, in which the head, forepaws, and hind legs were shielded by a lead jig, and animals were exposed to $15.5 \mathrm{~Gy}$. Nearly identical to the WBI experiments, while $100 \%$ of animals succumbed to radiotoxicity with denuded GI mucosa, $2 \mathrm{~A} 2$ increased 90 -day survival to $75 \%$ (Supplemental Figure 7; $P<0.01$ ).

These studies identify $2 \mathrm{~A} 2$ monoclonal antibody, the prototype in a new class of anti-ceramide therapeutics (21), as an effective countermeasure against the lethal radiation GI syndrome (schema 
A

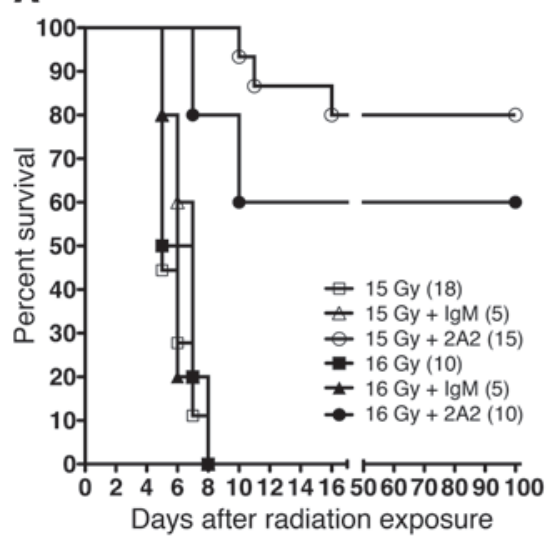

B

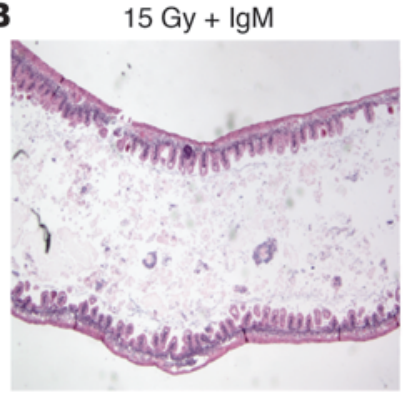

$15 \mathrm{~Gy}+2 \mathrm{~A} 2$

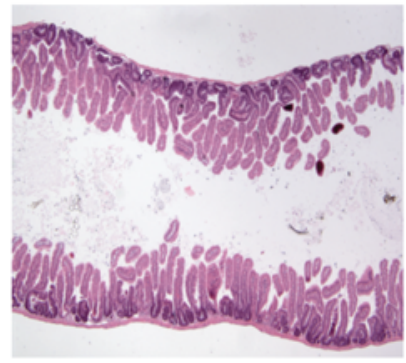

Figure 3

Purified monoclonal $2 \mathrm{~A} 2$ antibody protects against radiation-induced Gl lethality. (A) Intravenous 2A2 antibody injection 15 minutes before 15 Gy or 16 Gy WBI increases overall survival of mice administered HSCT $\left(3 \times 10^{6}\right.$ cells). The number of animals per group is in parenthesis. (B) Representative hematoxylin and eosin-stained sections of proximal jejunum harvested from agonal C57BL/6 mice 6 days after 15 Gy WBI plus IgM or 10 days after 15 Gy in a mouse administered 2A2 antibody 15 minutes before irradiation. Original magnification, $\times 100$.

tion and screened by ELISA. 2A2 IgM was purified from ascites using mannan-binding protein beads.

Cell culture. BAECs were cultured in DMEM, switched at confluency to DMEM plus $2 \%$ normal calf serum for 1-week minimum (8), and preincubated for 18 hours before irradiation in DMEM plus $0.2 \%$ human albumin.

Ceramide quantification. Diacylglycerol kinase assay was used for ceramide quantification (27).

ASMase activity. ASMase activity was quantified by radioenzymatic assay using $\left[{ }^{14} \mathrm{C}\right.$-methylcholine $]$ sphingomyelin

of 2A2 inhibition in Supplemental Figure 8). Previously published countermeasures are associated with clinically relevant toxicities or have displayed less efficacy when directly compared with 2A2. Basic fibroblast growth factor protects against radiation GI syndrome lethality (11) but is associated with serious hypotension $(22,23)$. The TLR5 agonist, CBLB502, is efficacious as a radioprotector of GI tract injury (24); however, reported data indicate that its effect is limited to lower radiation doses that are nonlethal to the GI tract (<13 Gy). Sphingosine-1-phosphate, while effective in protecting against radiation GI injury (25), was less effective than 2A2 in direct comparison (Supplemental Figure 9). Lastly, the angiopoietin derivative, COMP-Ang1 (26), was substantially less effective than $2 \mathrm{~A} 2$ as a radioprotector in our laboratory (data not shown). 2A2 antibody thus complements other promising agents under development, including CBLB502 from Cleveland BioLabs (24), which has been demonstrated to be effective at preventing BM lethality. As such, 2A2 antibody fulfills a mandate of the United States Department of Health and Human Services Public Health Emergency Medical Countermeasures Enterprise, a national security measure designed to protect the American public from the deliberate use of weapons of mass destruction, consistent with the goals of the president's Biodefense for the 21st Century and National Strategy for Medical Countermeasures against Weapons of Mass Destruction directives.

\section{Methods}

Antibody generation. BALB/c mice immunized by 3 intraperitoneal administrations of $2 \times 10^{7}$ human Kaposi's sarcoma (KS1767) cells at 1-week intervals were boosted 3 days prior to fusion. Harvested splenocytes were fused with mouse myeloma cells (P3X63Ag8.653; ATCC) at a 4:1 ratio using polyethylene glycol (MW $1,500 \mathrm{~g} / \mathrm{mol}$ ). Hybridomas were seeded at $1 \times 10^{5}$ cells per well in RPMI 1640 selection medium plus $20 \%$ fetal bovine serum, 10\% Hybridoma supplements (Sigma-Aldrich), $2 \mathrm{mM}$ L-glutamine, $100 \mathrm{U} / \mathrm{ml}$ penicillin, $100 \mu \mathrm{g} / \mathrm{ml}$ streptomycin, $10 \mathrm{mM}$ HEPES, and hypoxanthine-aminopterin-thymidine (Sigma-Aldrich). Supernatants were screened by FACS of KS1767 cells and ELISA using BSA-conjugated $\mathrm{C}_{16}$-ceramide. Selected hybridomas were subcloned 4 times by limited dilu-
(Amersham) (28).

Detection of CRPs. Confluent BAECs were detached with PBS plus $0.1 \%$ collagenase, $0.02 \% \mathrm{EDTA}$, and $0.5 \% \mathrm{BSA}$ at $37^{\circ} \mathrm{C}$ for 5 minutes, gently dispersed to obtain a single-cell suspension, and resuspended at $0.5 \times 10^{6}$ cells $/ \mathrm{ml}$ DMEM plus $0.2 \%$ human albumin. After irradiation, cells were incubated at $37^{\circ} \mathrm{C}$ and fixed with $2 \%$ paraformaldehyde for 15 minutes at $4^{\circ} \mathrm{C}$. Nonspecific sites were blocked with PBS containing $2 \%$ fetal bovine serum for 20 minutes, and cells were stained for surface ceramide using mouse monoclonal anti-ceramide antibody MID 15B4 IgM (1:50 dilution; Alexis Biochemicals) or for surface ASMase with rabbit polyclonal antiASMase AB 1598 (1:100 dilution) (16). Irrelevant mouse IgM or rabbit IgG served as isotype controls. After 3 washes with PBS plus $0.05 \%$ Tween-20, cells were stained for platforms with Texas Red-conjugated anti-mouse IgM or Cy3-conjugated anti-rabbit IgG (1:300 dilution, Roche), respectively, for 1 hour at $4^{\circ} \mathrm{C}$. Cells were mounted in fluorescent mounting medium (Dako). Fluorescence was detected using an Axiovert S-100 Zeiss microscope and SPOT digital camera. The percentage of cells containing platforms, i.e., those in which fluorescence condenses onto less than $25 \%$ of the cell surface, was determined in 150 to 250 cells/point.

Endothelial apoptosis. BAEC apoptosis was measured morphologically after Hoechst 33258 bis-benzimide staining (12). Small intestinal endothelial apoptosis was detected by double staining with TUNEL for apoptosis and a rat antibody against the endothelial cell surface marker CD31 (4).

Radiation source. Radiation was delivered to mice using a Shepherd Mark-I unit (model 68-SN643), operating a ${ }^{137}$ Cs source at 2.12 Gy per minute. For events occurring during the first 10 minutes, the dose rate was 13.1 Gy per minute.

Crypt microcolony survival assay. Crypt microcolony survival assay was performed according to Withers and Elkind $(11,20)$.

Mouse survival after WBI and designation of autopsy findings. Actuarial mouse survival was calculated by the Kaplan-Meier method (29). Terminally sick animals displaying agonal breathing were sacrificed by hypercapnia/ asphyxiation, and necropsy was performed to determine cause of death. Intestinal specimens, fixed in formaldehyde, were stained with hematoxylin (6). GI damage was diagnosed as cause of death when small intestines displayed denuded mucosa with nearly no villi or crypts or when mucosa displayed limited repair (6). 
Statistics. Significance was calculated using 2-tailed Student's $t$ test and Mantel log-rank test. $P<0.05$ was considered significant.

Study approval. Animal experimental protocols were approved by MSKCC Research Animal Resource Center.

\section{Acknowledgments}

This work was supported by NIH R01-CA085704 and MSKCC Experimental Therapeutics Center funded by William H. Goodwin and Alice Goodwin to R. Kolesnick, NIH R01-CA105125 to A. Haimovitz-Friedman, a gift from Virginia and D.K. Ludwig Fund for Cancer Research to Z. Fuks, and awards from AngelWorks, Gilson-Longenbaugh Foundation, and Marcus Foundation to W. Arap and R. Pasqualini. We thank Michelle Liew for assistance.

Received for publication July 26, 2011, and accepted in revised form February 15, 2012.

Address correspondence to: Richard Kolesnick, Sloan-Kettering Institute, 1275 York Avenue, New York, New York 10065, USA. Phone: 646.888.2174; Fax: 646.422.0281; E-mail: r-kolesnick@ski.mskcc.org.
1. Hendry JH, Potten CS, Roberts NP. The gastrointestinal syndrome and mucosal clonogenic cells: relationships between target cell sensitivities, LD50 and cell survival, and their modification by antibiotics. Radiat Res. 1983;96(1):100-112.

2. Hendry JH, Roberts SA, Potten CS. The clonogen content of murine intestinal crypts: dependence on radiation dose used in its determination. Radiat Res. 1992;132(1):115-119.

3. Potten CS. A comprehensive study of the radiobiological response of the murine (BDF1) small intestine. Int J Radiat Biol. 1990;58(6):925-973.

4. Paris F, et al. Endothelial apoptosis as the primary lesion initiating intestinal radiation damage in mice. Science. 2001;293(5528):293-297.

5. Rotolo JA, Kolesnick R, Fuks Z. Timing of lethality from gastrointestinal syndrome in mice revisited. Int J Radiat Oncol Biol Pbys. 2009;73(1):6-8.

6. Rotolo JA, et al. Bax and Bak do not exhibit functional redundancy in mediating radiation-induced endothelial apoptosis in the intestinal mucosa. Int J Radiat Oncol Biol Phys. 2008;70(3):804-815.

7. Stancevic B, Kolesnick R. Ceramide-rich platforms in transmembrane signaling. FEBS Lett. 2010; 584(9):1728-1740

8. Truman JP, et al. Endothelial membrane remodeling is obligate for anti-angiogenic radiosensitization during tumor radiosurgery. PLoS One. 2010;5(9).

9. Marathe S, et al. Human vascular endothelial cells are a rich and regulatable source of secretory sphingomyelinase. Implications for early atherogenesis and ceramide-mediated cell signaling. J Biol Chem. 1998;273(7):4081-4088.

10. Santana $\mathrm{P}$, et al. Acid sphingomyelinase-deficient human lymphoblasts and mice are defective in radi- ation-induced apoptosis. Cell. 1996;86(2):189-199.

11. Maj JG, Paris F, Haimovitz-Friedman A, Venkatraman E, Kolesnick R, Fuks Z. Microvascular function regulates intestinal crypt response to radiation. Cancer Res. 2003;63(15):4338-4341.

12. Haimovitz-Friedman A, et al. Ionizing radiation acts on cellular membranes to generate ceramide and initiate apoptosis. J Exp Med. 1994;180(2):525-535.

13. Verheij M, et al. Requirement for ceramide-initiated SAPK/JNK signalling in stress-induced apoptosis. Nature. 1996;380(6569):75-79.

14. Liao WC, et al. Ataxia telangiectasia-mutated gene product inhibits DNA damage-induced apoptosis via ceramide synthase. J Biol Chem. 1999; 274(25):17908-17917.

15. Fuks $Z$, et al. Basic fibroblast growth factor protects endothelial cells against radiation-induced programmed cell death in vitro and in vivo. Cancer Res. 1994;54(10):2582-2590.

16. Rotolo JA, Zhang J, Donepudi M, Lee H, Fuks Z, Kolesnick R. Caspase-dependent and -independent activation of acid sphingomyelinase signaling. J Biol Chem. 2005;280(28):26425-26434.

17. Grassmé $\mathrm{H}$, et al. CD95 signaling via ceramiderich membrane rafts. J Biol Chem. 2001; 276(23):20589-20596.

18. Grassmé H, et al. Host defense against Pseudomonas aeruginosa requires ceramide-rich membrane rafts. Nat Med. 2003;9(3):322-330.

19. Göggel R, et al. PAF-mediated pulmonary edema: a new role for acid sphingomyelinase and ceramide. Nat Med. 2004;10(2):155-160.

20. Withers HR, Elkind MM. Microcolony survival assay for cells of mouse intestinal mucosa exposed to radiation. Int J Radiat Biol Relat Stud Phys Chem
Med. 1970;17(3):261-267.

21. Rotolo JA, Kolesnick R, Pasqualini R, Arap W, inventors; Sloan Kettering Institute For Cancer Research, assignee. Methods for treating and preventing GI syndrome and graft versus host disease. US patent 12/599,280. May 6, 2008.

22. Bush MA, et al. Pharmacokinetics and pharmacodynamics of recombinant FGF-2 in a phase I trial in coronary artery disease. J Clin Pharmacol. 2001; 41(4):378-385.

23. Simons M, et al. Pharmacological treatment of coronary artery disease with recombinant fibroblast growth factor-2: double-blind, randomized, controlled clinical trial. Circulation. 2002;105(7):788-793.

24. Burdelya LG, et al. An agonist of toll-like receptor 5 has radioprotective activity in mouse and primate models. Science. 2008;320(5873):226-230.

25. Bonnaud S, et al. Sphingosine-1-phosphate activates the AKT pathway to protect small intestines from radiation-induced endothelial apoptosis. Cancer Res. 2010;70(23):9905-9915.

26. Cho $\mathrm{CH}$, et al. Designed angiopoietin-1 variant, COMP-Ang1, protects against radiation-induced endothelial cell apoptosis. Proc Natl Acad Sci U S A. 2004;101(15):5553-5558.

27. Garzotto $M$, et al. Reversal of radiation resistance in LNCaP cells by targeting apoptosis through ceramide synthase. Cancer Res. 1999;59(20):5194-5201.

28. Schissel SL, Schuchman EH, Williams KJ, Tabas I. $\mathrm{Zn}^{2+}$-stimulated sphingomyelinase is secreted by many cell types and is a product of the acid sphingomyelinase gene. J Biol Chem. 1996;271(31):18431-18436.

29. Kaplan EL, et al. Nonparametic estimation from incomplete observations. J Am Stat Assoc. 1958; 53(282):457-481. 